\title{
Time-resolved Element-selective Probing of Charge Carriers in Solar Materials
}

\author{
James Budarz ${ }^{a}$, Fabio G. Santomauroa, M. Hannelore Rittmann-Frank ${ }^{a}$, Chris J. Milne ${ }^{b}$, Thomas \\ Huthwelker ${ }^{c}$, Daniel Grolimund ${ }^{c}$, Jochen Rittmann ${ }^{b}$, Dominik Kinschel ${ }^{a}$, Thomas Rossi ${ }^{a}$, and Majed \\ Chergui $^{\mathrm{a}}$
}

\begin{abstract}
We review our recent results on the implementation of picosecond (ps) X-ray absorption spectroscopy to probe the electronic and geometric structure of centres formed by photoexcitation of solar materials such as $\mathrm{TiO}_{2}$ polymorphs and inorganic $\mathrm{Cs}$-based perovskites. The results show electron localization at $\mathrm{Ti}$ defects in $\mathrm{TiO}_{2}$ anatase and rutile and small hole polaron formation in the valence band of $\mathrm{CsPbBr}_{3}$, all within 80 ps. This method is promising for the study of the ultrafast time scales of such processes, especially with the advent of the Swiss X-ray Free Electron Laser (SwissFEL).
\end{abstract}

Keywords: Perovskites $\cdot$ Solar materials $\cdot \mathrm{TiO}_{2} \cdot$ Ultrafast $\cdot$ X-ray absorption spectroscopy

\section{Introduction}

A new class of solid-state solar cells based on $\mathrm{TiO}_{2}$ operating with organic perovskite sensitizers has been under intense investigation in recent years. Organic Lead Halide Perovskites (LHPs) have the $\mathrm{APb}_{3}$ stoichiometry, where $A$ is an organic cation $\left(\mathrm{CH}_{3} \mathrm{NH}_{3}^{+}, \mathrm{HC}\left(\mathrm{NH}_{2}\right)_{2}{ }^{+}\right.$or others $)$and $\mathrm{X}$ is a halide anion $\left(\mathrm{I}^{-}, \mathrm{Br}^{-}\right.$, or $\left.\mathrm{Cl}^{-}\right)$. The LHPbased generation of solid-state solar cells have reached efficiencies exceeding $20 \%$ within only a few years of study.[1] These materials benefit from tunability of optical absorption (band gap) via halide substitution, a better match with the solar spectrum, ease of synthesis and application to fully-solid state solar cells (unlike the early dye-sensitized solar cells, DSSC). LHPs exhibit many features that make them effective and practical sensitizers, such as efficient generation of free charges, long charge carrier diffusion length, ${ }^{[2]}$ and low charge carrier recombination rates.

\footnotetext{
${ }^{*}$ Correspondence: Prof. Dr. M. Chergui ${ }^{a}$ E-mail: Majed.chergui@epfl.ch

${ }^{a}$ Ecole Polytechnique Fédérale de Lausanne Laboratoire de Spectroscopie Ultrarapide (LSU) and Lausanne Centre for Ultrafast Science (LACUS) ISIC, FSB, CH-1015 Lausanne 'SwissFEL, Paul Scherrer Institut $\mathrm{CH}-5232$ Villigen PSI

'Swiss Light Source, Paul Scherrer Institut $\mathrm{CH}-5232$ Villigen PSI
}

However, several critical factors and properties still limit the widespread implementation of organic LHP-based solar cells. They lack high carrier mobility, ${ }^{[3,4]}$ long-term stability and are sensitive to water. A promising alternative has arisen wherein caesium replaces the organic cation, partially or completely. ${ }^{[5,6]}$ This substitution promises to improve the lifespan of the material and makes it significantly more resistant to irradiation. $\mathrm{CsPbX}_{3}$ is expected to exhibit identical charge carrier behaviour to organic LHP because neither the conduction band $(\mathrm{CB})$ nor the valence band (VB) have been calculated to appear at similar energy to the caesium orbitals in the material.[6]

A similar architecture to DSSCs can be made from the combination of LHP and transition metal oxides (TMO), such as $\mathrm{TiO}_{2}$, also called perovskite sensitized solar cells (PSSC). However, LHPs have been shown to generate electricity without the TMO substrate, ${ }^{[7]}$ which questions the role of the latter and even the existence of an electron injection in its presence. Electron injection from LHPs to $\mathrm{TiO}_{2}$ has been convincingly concluded on the basis of optical pump/THz probe in comparative studies with $\mathrm{LHP}$ on $\mathrm{Al}_{2} \mathrm{O}_{3}{ }^{\left[{ }^{8]}\right.}$ However, the use of two different substrates can introduce somewhat different responses in that spectral range, especially at the interface, which may not be relevant to the injection. One needs therefore to resort to elementor substrate-specific methods.

In recent years, we implemented time-resolved X-ray absorption spectroscopy (XAS) at synchrotrons as a novel tool to probe the fate of the electrons in TMOs. In a study of bare and ruthenium-sensitized anatase $\mathrm{TiO}_{2}$ nanoparticles (NPs) in solution, we monitored the response of the system upon electron delivery to the conduction band (CB) of the TMO either by direct band-gap excitation or upon visible excitation of the Ru-dye. ${ }^{[9]}$ We also probed the $\mathrm{Ru} \mathrm{L}$ edge of the dye and detected its change of oxidation state upon electron transfer to the TMO. The transient spectra measured at the Ti K-edge reflected trapping of the electron at pre-existing defects in the TMO, providing an unambiguous signature of electron injection. [10]

In order to implement this approach in the case of PSSCs, the first step is to investigate the response of the different constituents of the solar cell upon photoexcitation: the sensitizer (here the LHP) and the TMO, at the X-ray absorption edges of the atoms forming these materials. In this contribution, building on our previous work on anatase $\mathrm{TiO}_{2},{ }^{[9,10]}$ we report time-resolved XAS studies of photoexcited rutile $\mathrm{TiO}_{2}$ NPs. Further, we investigated photoexcited inorganic Cs-based perovskite NPs, establishing the response of each of the atomic constituents $(\mathrm{Cs}, \mathrm{Pb}, \mathrm{Br})$ of these materials. These results demonstrate that it is possible to interrogate each element in the PSSCs with femtosecond to picosecond time resolution.

\section{X-ray Absorption Spectroscopy}

X-ray Absorption Spectroscopy (XAS) provides information about the immediate environment and electronic structure of specific chemical elements by tuning the monochromatic X-rays to the corresponding absorption edges. Specifically, the pre-edge region of a spectrum contains bound-bound transitions from the core-orbital to the valence orbitals of the atoms, which are those making the chem- 
ical bonds. These are the orbitals that are modified upon photoexcitation and therefore interrogating them in an optical pump/X-ray probe experiment delivers information about their occupancy. The edge itself represents the ionization threshold of the atom and it is very sensitive to its oxidation state. Up to about $50 \mathrm{eV}$ above the edge is the so-called X-ray absorption near-edge spectrum (XANES), which contains modulations due to the multiple scattering of the X-rays-generated photoelectron with the neighbouring atoms. It therefore reflects the 3D structure of the environment around the atom of interest. At even higher energies lies the extended X-ray absorption fine structure (EXAFS) region, whose modulations reflect the single scattering events of the photoelectron with the neighbouring atoms. It therefore also contains information about the local geometry, mainly bond distances and coordination numbers. Because EXAFS is due to single scattering events, it is much simpler to model than XANES, and for that reason, it has been more popular in probing the local structure around specific centres in materials, molecules and proteins. ${ }^{[11]}$

The implementation of time domain XAS at synchrotrons has been carried out over the last 15 years or so. ${ }^{[12-14]}$ One important recent development has been the optical pump/X-ray spectroscopy probe scheme at $\mathrm{MHz}$ repetition rates. ${ }^{[15]}$ Combined with a sample delivery, in the form of a liquid jet, that allows continuous refreshing of the sample and thus minimizes sample heating and damage, this pump-probe scheme has been used to probe structural and electronic changes in molecules, ${ }^{[14]}$ proteins $^{[16]}$ and nanoparticles in solution.[14]

Briefly, the picosecond pump laser system operates at half the repetition rate of the synchrotron source that delivers an isolated (so-called 'camshaft') X-ray pulse at $1.04 \mathrm{MHz}$ at the Swiss Light Source (SLS). The laser is synchronized to the storage ring via the radiofrequency (RF) signal of the synchrotron master clock. The transient signal consists of the difference between the X-ray absorption of the photoexcited sample minus that of the unexcited sample, averaged over several laser/X-ray pairs of pulses at a given time delay between the pump and the probe pulses. Details of the data acquisition procedure are given in ref. [15]. This experimental technique has been implemented as a mobile setup at the microXAS, PHOENIX and SuperXAS beamlines of the Swiss Light Source (SLS)

\section{Electron Trapping in Anatase and Rutile $\mathrm{TiO}_{2}$}

Fig. 1 shows the steady state Ti K-edge XANES spectra of amorphous, anatase and rutile titania nanoparticles (NPs) with typical diameters of 10 to $20 \mathrm{~nm}$. The former two spectra are similar to those presented in ref. [9]. Here we briefly recall the main results from this study. The pre-edge XAS region (Fig. 1) exhibits four peaks labelled $\mathrm{A} 1-\mathrm{A} 3$ and $\mathrm{B}$. The $\mathrm{A} 2$ and $\mathrm{A} 3$ peaks are separated by $\sim 1 \mathrm{eV}$, relative to the $\mathrm{A} 1$ and B peaks. In amorphous NPs, they appear stronger as also reported in refs $[17,18]$. These peaks are typical of K-shell spectra, ${ }^{[11]}$ i.e. transitions from the $1 \mathrm{~s}$ core orbital to unoccupied Ti $3 \mathrm{~d}$ valence orbitals. In the regular octahedral $\mathrm{Ti}^{4+}\left(3 \mathrm{~d}^{0}\right)$ hexacoordinated sites of bulk anatase, these transitions have a quadrupole character, but anatase $\mathrm{TiO}_{2}$ presents a weak $\mathrm{D}_{2 \mathrm{~d}}$ distortion that makes them partially allowed by virtue of $3 d / 4 p$ mixing, which introduces a dipole component.[19,20] Stronger deviations from octahedral symmetry would further enhance such mixings. The exact assignment of these peaks is still a matter of debate, but there is consensus that $\mathrm{A} 1$ has a predominantly quadrupole character, A2 and A 3 have a quadrupole + dipole character, while B is predominantly a dipole transition. ${ }^{[9]}$ The pre-edge region of the rutile NPs exhibits only three peaks $\mathrm{A} 1, \mathrm{~A} 3$ and $\mathrm{B}$, as previously reported. ${ }^{[17]}$ Strong O 2p-Ti $3 \mathrm{~d}$ hybridization gives to A3 and B peaks predominantly dipole character, whereas A1 is predominantly quadrupolar as in the anatase case. Other strong features (C, D1, D2, E), at or above the edge (Fig. 1) have principally a dipole character.[21] The spectral differences between anatase, rutile and amorphous NPs suggest different coordination geometries around the $\mathrm{Ti}$ atoms. ${ }^{[22]}$ As mentioned above, the A2 peak has been shown to have a surface character, ${ }^{[17]}$ but its pronounced presence in bulk amorphous samples may suggest a more specific explanation for its enhanced intensity at the surface. Above the edge, the absorption spectra of anatase and rutile are, as expected, clearly more structured than that of amorphous NPs. This, along with the very different powder diffraction patterns of the three forms, ${ }^{[22]}$ point to both short and long range disorder in the amorphous case. The studies by Luca and co-workers also show that anatase $\mathrm{TiO}_{2}$ NPs have an ordered core with a defect-rich surface shell containing a high degree of Ti under-coordination. ${ }^{[17,22]}$ For rutile $\mathrm{TiO}_{2}$ NPs, Luca and co-workers did

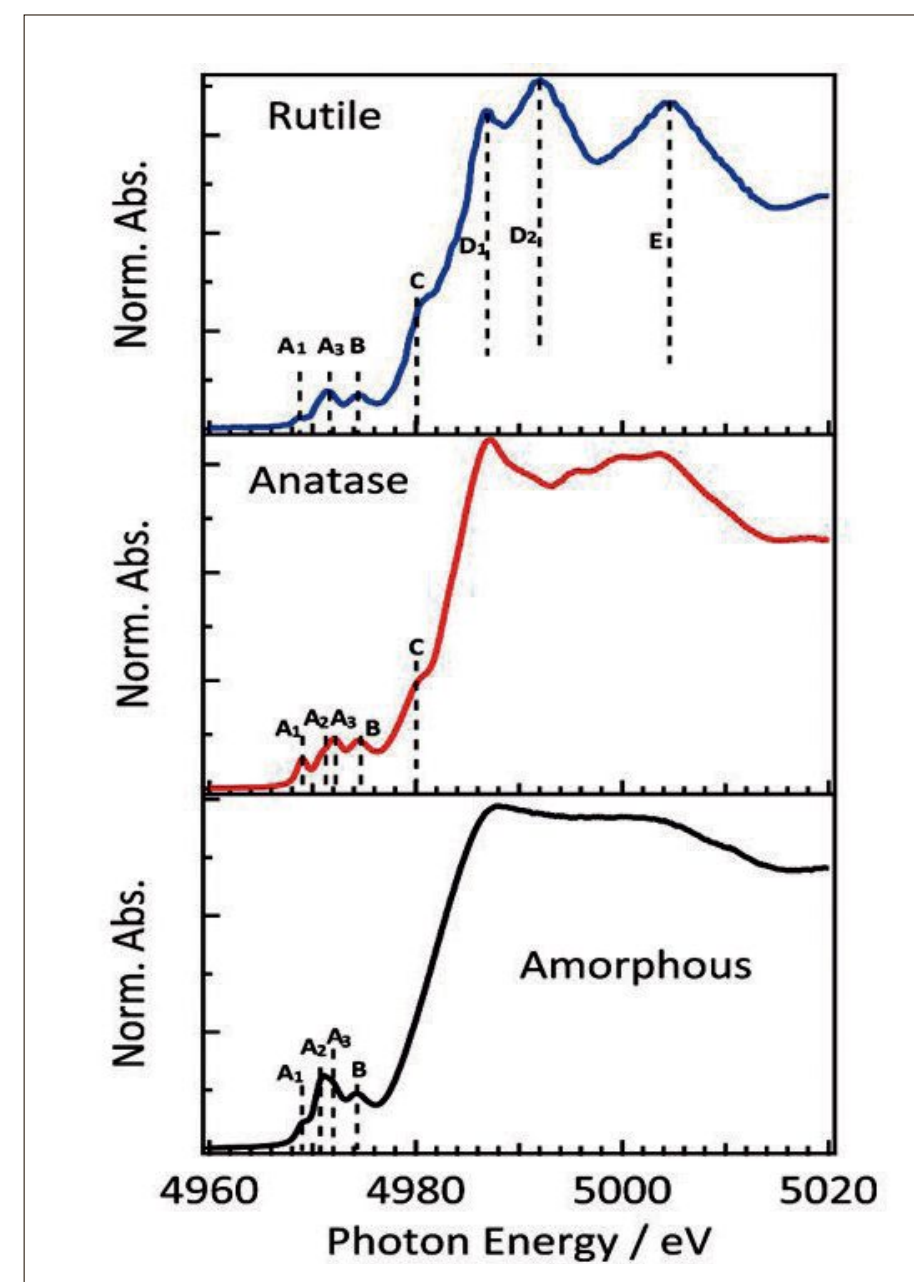

Fig. 1. Steady state Ti K-edge absorption near edge structure (XANES) of rutile, anatase and amorphous $\mathrm{TiO}_{2} \mathrm{NPs}$ in solution. The dotted vertical lines highlight the main features in the pre-edge region $\left(A_{1}, A_{2}, A_{3}, B\right.$ and C) and above the edge $\left(D_{1}, D_{2}\right.$ and $\left.E\right)$. The absence of the $A$ feature in case of rutile NPs can be seen and is discussed in ref. [17]. 
not detect defects, probably due to the fact that they are less frequent.

As mentioned before, we demonstrated for the first time optical pump/X-ray absorption probe spectroscopy with 80 ps time resolution upon excitation of bare (at $355 \mathrm{~nm}$ ) and ruthenium dye-sensitized (RuN719) 10-20 nm diameter anatase and amorphous NPs (at $532 \mathrm{~nm}$ ). ${ }^{[9]}$ The transient in the case of bare anatase $\mathrm{TiO}_{2} \mathrm{NPs}_{\mathrm{s}}$ is shown in Fig. 2a. It can be well reproduced by taking the difference of the static amorphous spectrum shifted by $-1 \mathrm{eV}$ minus the static anatase spectrum (Fig. 1) yielding the dashed trace in Fig. 2a (the scaling was done by normalizing the static difference and the transient spectra at the maximum with a factor of 0.016). This implies that rather than an unlikely structural deformation from ordered (anatase) to disordered (amorphous), there is a shift of spectral weight from regular sites to disordered ones with the generated electrons getting trapped at pre-existing defects and acting as a 'contrast agent' to reveal them. We argued that the photogenerated electrons reduce with nearly a full electron charge $\mathrm{Ti}^{4+}$ at penta-coordinated defect sites, forming $\mathrm{Ti}^{3+}$ centres. Because the density of traps is highest in the surface shell region, it is likely that the observed trapping occurs mostly therein. In the case of amorphous NPs, reduced $\mathrm{Ti}^{3+}$ centres are also formed as a result of photoexcitation, but the trapping can, a priori, occur anywhere in the NP. In the case of injection of electrons, we found that defect $\mathrm{Ti}^{3+}$ traps also form with a full electron charge on them but they are located at the outer surface of the NP. ${ }^{9}$

The transient XAS of rutile $\mathrm{TiO}_{2}$ NPs recorded $100 \mathrm{ps}$ after $355 \mathrm{~nm}$ excitation is shown in Fig. 2b. Significant changes are observed in the pre-edge, the edge and above-edge regions reflecting substantial photoinduced modifications of both the electronic and the geometric structure of Ti centres. The transient XAS is quite different to that observed for anatase $\mathrm{TiO}_{2}$. However, when using the same approach as for anatase and taking the difference of the amorphous spectrum shifted down in energy by 0.5 to $1 \mathrm{eV}$ minus the rutile ground state spectrum, we obtain the dashed trace using a scaling factor of about 0.003 , which agrees fairly well with the experimental rutile transient (Fig. 2b). This allows us to conclude that just as in anatase $\mathrm{TiO}_{2}$, the electrons transferred to the $\mathrm{CB}$ in rutile $\mathrm{TiO}_{2}$ NPs end up trapped at pre-existing defects, which are identical to those of anatase $\mathrm{TiO}_{2}$. This is not too surprising as the difference between these two polymorphs does not concern the first shell of $\mathrm{O}$ neighbours around $\mathrm{Ti}$, but the second. Since we argued that the trapping sites are pentacoordinated ones, it is likely that they are identical in both cases.
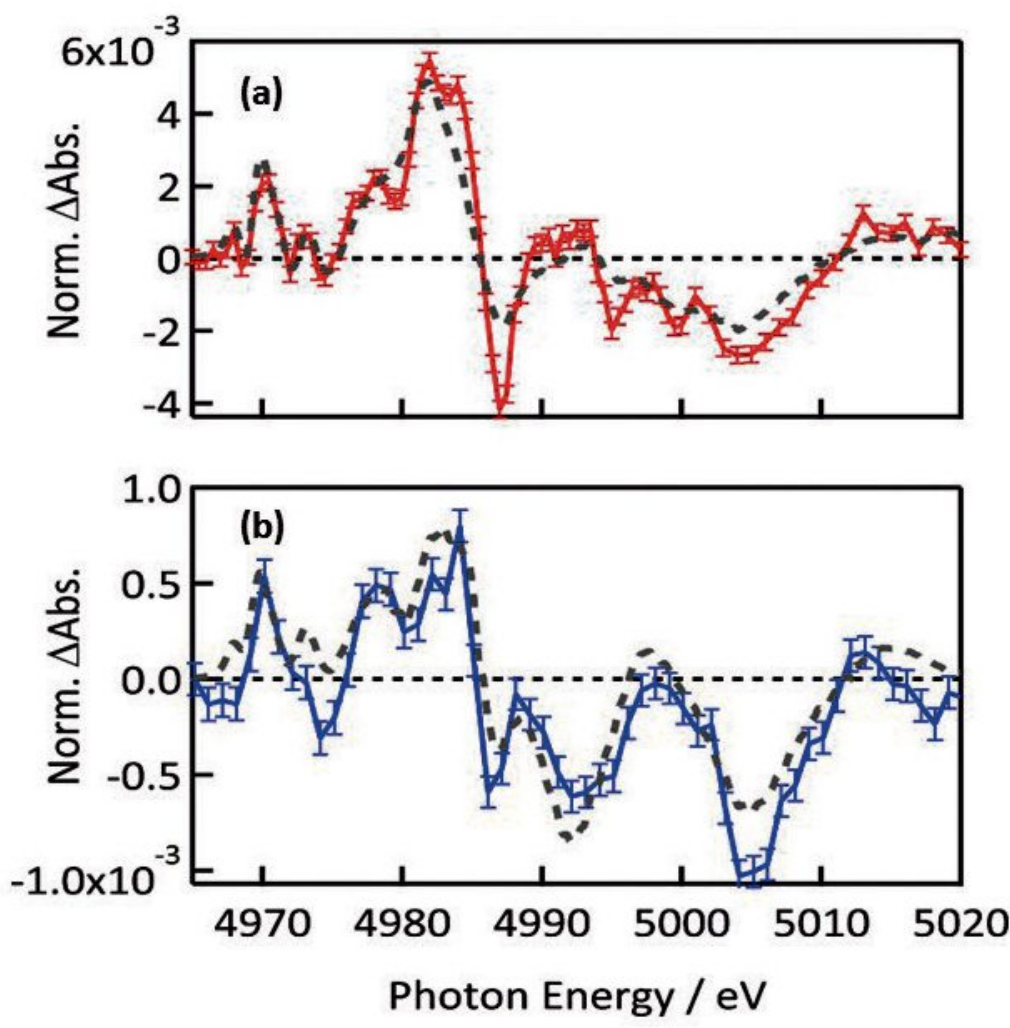

Fig. 2. Transient Ti K-edge absorption spectra at 100 ps time delay of: (a) anatase NPs (red curve) along with the calculated difference spectrum of the $1 \mathrm{eV}$ red shifted amorphous steady state spectrum minus the steady state anatase spectrum (dotted grey curve, taken from ref. [9]). (b) Rutile NPs (blue curve) along with the calculated difference spectrum of the $1 \mathrm{eV}$ red shifted amorphous steady state spectrum minus the steady state rutile spectrum (dotted grey curve). In both cases, the excitation wavelength was $355 \mathrm{~nm}$.

The scaling factor used in Fig. $2 b$ is about five times lower for than for Fig. 2a $(0.3 \%$ vs $1.6 \%)$, although we expect a slightly higher excitation yield for rutile considering that the excitation fluence is the same and the concentration was set to have an optical density of 1.2 at $355 \mathrm{~nm}$ in both cases. Thus, the lower scaling factor in rutile must be attributed to a lower probability of electron trapping, which is in line with the conclusion that the concentration of defects is lower.[17]

The time traces of the two types of NPs were also recorded. For bare anatase NPs, they were best fitted using bi-exponential functions convoluted with the instrument response function (about $80 \mathrm{ps}$ ). This yielded time constants of 0.3 and $6 \mathrm{~ns}$, which were similar in the pre-edge (4.979 $\mathrm{keV})$, the edge (4.982 keV) and the postedge $(5.15 \mathrm{keV})$, within error bars, indicating that the photoinduced electronic and structural changes originate from the same species. In the case of rutile, the kinetic behaviour is somewhat different and the trace can be fitted using either a monoexponential decay (with a time constant of $1.4 \mathrm{~ns}$ ) or a biexponential decay ( 0.5 and $36 \mathrm{~ns})$. However, the limited range of the time trace and the poor $\mathrm{S} / \mathrm{N}$ make us cautious about these values. Further experi- ments at X-ray free electron lasers, such as SwissFEL, will greatly increase the $\mathrm{S} / \mathrm{N}$ and allow femtosecond resolution, which delivers more insight into the structural response, as recently shown in a study by Obara et al. ${ }^{[23]}$ Indeed, following on the work by Santomauro et al. ${ }^{[10]}$ on electron trapping in $\mathrm{TiO}_{2}$, they found that the response of the edge shift (due to the change of oxidation state) was faster than that of the structural features, implying that the structural rearrangement at the defects responds to the electronic structure changes.

\section{Charge Carriers in Caesium-based Perovskites}

Next, we investigated the fate of charge carriers in photoexcited $\mathrm{CsPbBr}_{3}$ and $\mathrm{CsPb}(\mathrm{ClBr})_{3} \mathrm{NPs}$ in solution as a foregoing step for the probing of PSSCs. We summarize here our recently published results using picosecond XAS ${ }^{24]}$ at the bromine $\mathrm{K}$ edge, the lead $\mathrm{L}_{3}$ edge, and the caesium $\mathrm{L}_{2}$ edge. The constituents of Cs-based inorganic perovskites have the following oxidation state (electronic configuration): $\mathrm{Br}^{-}\left(\ldots 4 \mathrm{p}^{6}\right), \mathrm{Cs}^{+}\left(\ldots 5 \mathrm{p}^{6} 6 \mathrm{~s}^{0}\right)$ and $\mathrm{Pb}^{2+}$ $\left(\ldots 5 \mathrm{~d}^{10} 6 \mathrm{~s}^{2} 6 \mathrm{p}^{0}\right)$. The valence band (VB) is mostly dominated by the $\mathrm{Br} 4 \mathrm{p}$-orbitals hy- 
bridized with $\mathrm{Pb}$ 6s-orbitals, while the $\mathrm{CB}$ is dominated by $\mathrm{Pb} 6 \mathrm{p}$-orbitals. ${ }^{[5,6]}$

Fig. 3 shows the static $\mathrm{Br} \mathrm{K}$-edge of $\mathrm{CsPbBr}_{3} \mathrm{NPs}$ (it is identical in the case of $\mathrm{CsPb}(\mathrm{ClBr})_{3}$ ) along with the transients recorded in $100 \mathrm{ps}$ transient spectra of $\mathrm{CsPbBr}_{3}$ (red) and $\mathrm{CsPb}(\mathrm{ClBr})_{3}$ (blue). The static spectrum resembles closely the XAS spectrum of aqueous bromide. ${ }^{[25]}$ Likewise, the transients are typical of those obtained for aqueous bromide ${ }^{[25]}$ or iodide ${ }^{[26,27]}$ turning to bromine or iodine, respectively, after photoexcitation, namely: a) the appearance of a pre-edge transition (here at 13.475 $\mathrm{keV}$ ) due to the s-p transition that becomes possible after a hole is created in the $4 p$ orbital; b) a blue shift of the edge which gives rise to a first derivative-like shape of the transient between 13.477 and 13.49 $\mathrm{keV}$. In the context of the perovskites, this means a change from $\mathrm{Br}^{-}$to $\mathrm{Br}^{0}$ upon photoexcitation, which reflects localization of a near-full hole charge at the halogen. The above edge region in the transient has clearly modulated features, suggesting formation of a new structure around the localised hole, that we argued to be due to a small polaron. ${ }^{[24]}$

Fig. 4 shows the static $\mathrm{Pb} \mathrm{L}_{3}$-edge, which is broad and unstructured, as is often the case with heavy atoms. The transients at 100 ps time delay are also displayed. Although the S/N ratio is low, some general trends can be identified for both compounds: a positive feature appears in the pre-edge region (it is clearer in $\mathrm{Cs} \mathrm{PbBr}_{3}$ ), while a minimum shows up right at the beginning of the above-edge region. At the $\mathrm{L}_{3}$-edge we probe $2 \mathrm{p}_{3 / 2} \rightarrow \mathrm{nd}, \mathrm{n} \geq 6$ transitions, therefore the $6 \mathrm{p}$ orbitals that make up the $\mathrm{CB}$ are in principle not accessible and most of the edge absorption is due to transitions to the $\mathrm{Pb} 6 \mathrm{~d}$ orbitals. In a study of the lead(II)-oxide and -sulphide compounds, Swarbrick et al. ${ }^{[28]}$ showed, however, that the $\mathrm{Pb} \mathrm{L}_{3}$-edge spectra also contain contributions of $\mathrm{Pb} \mathrm{s}$ and p-orbitals. Especially, the latter appear as weak contributions in the pre-edge region as a result of $\mathrm{p}$ - $\mathrm{d}$ mixing. An alternative explanation for the occurrence of the positive pre-edge features in the transients is hybridization of $\mathrm{Pb} 6 \mathrm{~s}-\mathrm{Br} 4 \mathrm{p}$ orbitals. The depletion of the $\mathrm{Br} 4 \mathrm{p}$ orbitals implies that of the $\mathrm{Pb} 6 \mathrm{~s}$ orbitals, which means that a partial transition from the $\mathrm{Br} 2 \mathrm{p}_{3 / 2}$ core orbital may become possible. Both effects reflect an enhancement of the transition probability in the pre-edge region and may be concurrent. Further studies are on-going to identify which of the two contributions is dominant.

The edge-region corresponds to the Fermi level and therefore an excess of electrons in the CB should lead to a decrease of intensity in the above edge region, which is actually the case in the transients. This

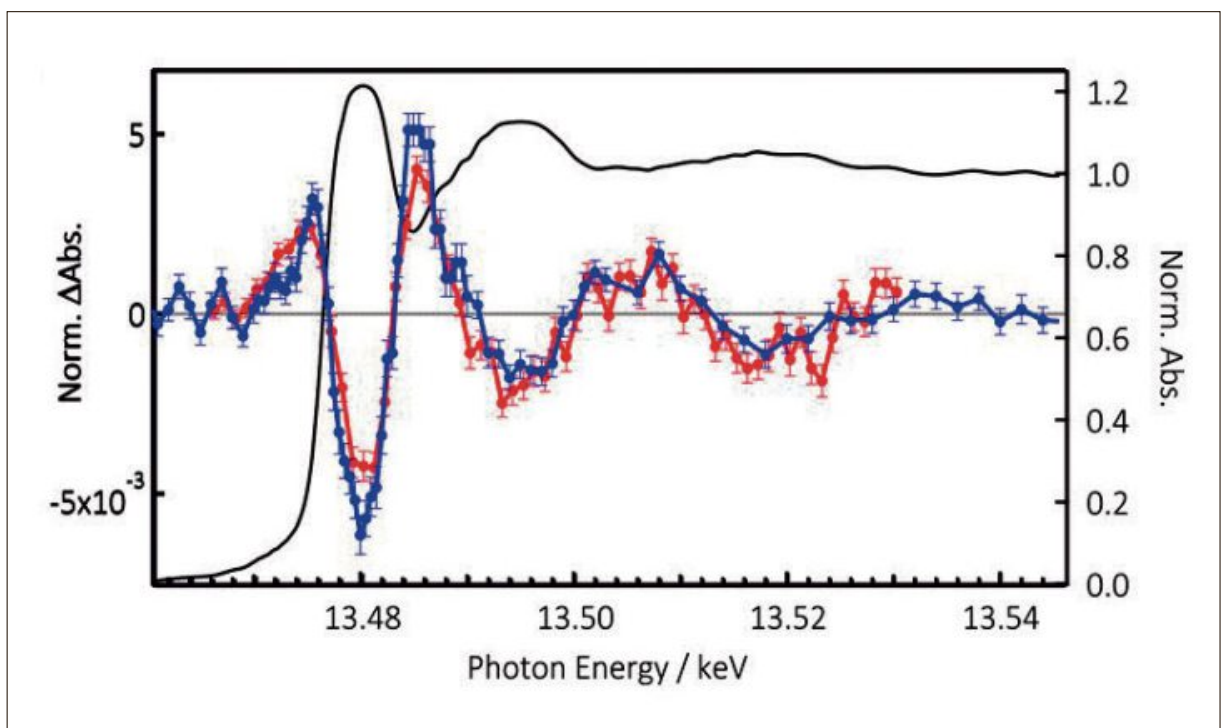

Fig. 3. $\mathrm{Br}$ K-edge absorption spectrum of $\mathrm{CsPb}(\mathrm{ClBr})_{3}$ (black trace) with transients at $100 \mathrm{ps}$ of $\mathrm{CsPb}(\mathrm{ClBr})_{3}$ (blue trace) and $\mathrm{CsPbBr}_{3}$ (red trace) nanocrystals in solution excited at $355 \mathrm{~nm}$. Reproduced from ref. [24].

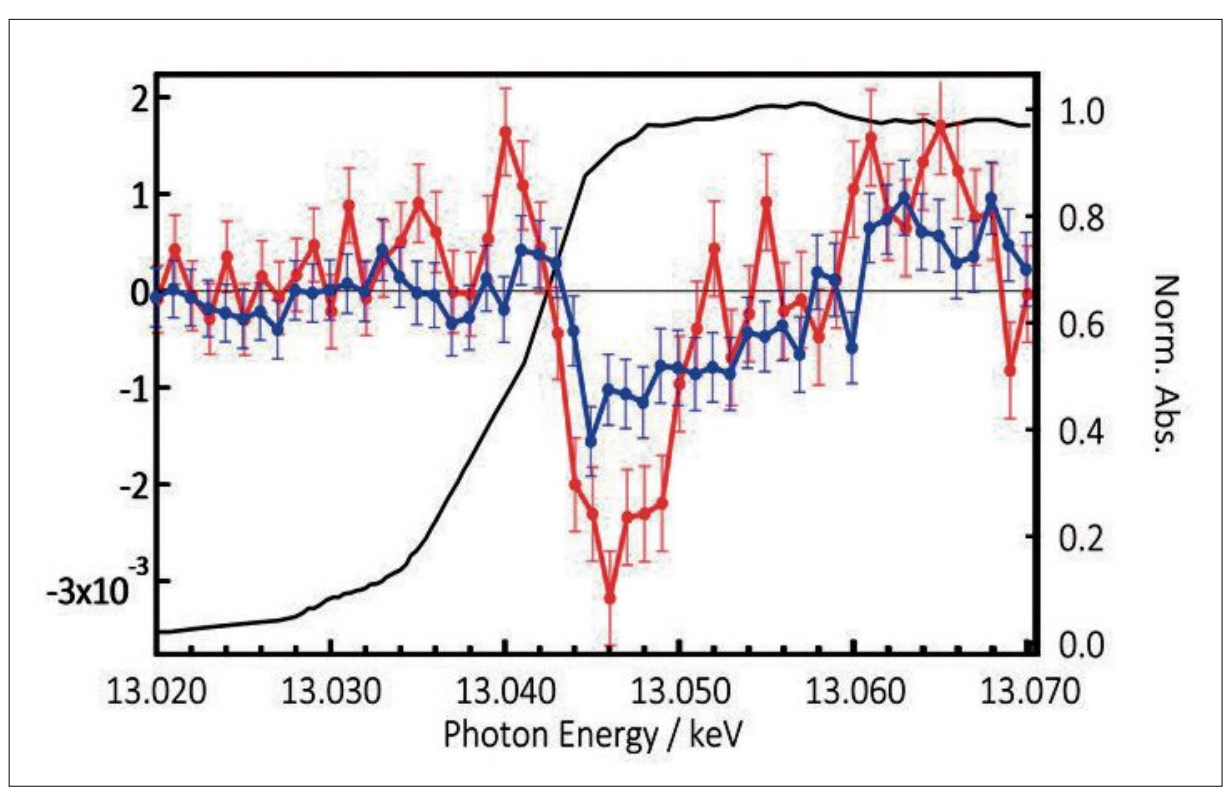

Fig. 4. Steady-state $\mathrm{Pb} \mathrm{L}_{3}$-edge absorption spectrum of $\mathrm{CsPb}(\mathrm{ClBr})_{3}$ nanocrystals (black trace) and transient spectra at $100 \mathrm{ps}$ time delay after photoexcitation at $355 \mathrm{~nm}$ of $\mathrm{CsPb}(\mathrm{ClBr})_{3}$ (blue trace) and $\mathrm{CsPbBr}_{3}$ (red trace).

is akin to phase space filling reported in optical studies of semiconductors, ${ }^{[29]}$ and it suggests that electrons are free carriers in the CB. Further confirmation of the present observations is given by the recent ps XAS studies by Zheng et al. ${ }^{[30]}$ on $\mathrm{CH}_{3} \mathrm{NH}_{3} \mathrm{PbBr}_{3}$ nanoparticles. They report a similar decrease of absorption around $13.045 \mathrm{keV}$ at 100-150 ps time delay. They extended their scans into the EXAFS region and attributed the changes therein to bond elongation of $\mathrm{Pb}-\mathrm{Br}$ bonds by $\sim 0.03 \AA$, although this conclusion contradicts our conclusions of electrons as free carriers in the CB.

We also probed the caesium $\mathrm{L}_{2}$-edge and found no change after photoexcitation, which is expected since the counter ion orbitals lie at much higher energies than those of $\mathrm{Pb}^{[5]}$ Thus either they do not participate in the electronic dynamics or, most likely, the latter are over at the 80 ps time delay at which we probe the sample. At this stage we conclude from our results that delocalised electrons occur in the $\mathrm{CB}$ while holes are localised as small polarons at $\mathrm{Br}$ atoms in the $\mathrm{VB}$.

\section{Conclusion}

This short review of our time-resolved $\mathrm{X}$-ray spectroscopic studies was aimed at stressing the advantages of an element-selective, time-resolved probe of photoex- 
cited solar materials. We have mainly focussed on X-ray absorption, which probes the density of unoccupied states, however the combination with X-ray emission, which probes the density of occupied states should deliver an additional degree of insight into the fate of charge carriers in such materials. A good example is our recent work on $\mathrm{ZnO}$ where for the first time holes are visualized in photoexcited transition metal oxides. [31]

Further developments of the studies presented herein would be to investigate a PSSC (i.e. the perovskites in contact with $\mathrm{SnO}_{2} / \mathrm{TiO}_{2}$ ). However, this calls for a special preparation of the samples, which is under development at present. While X-ray spectroscopic methods provide an unambiguous signature of the fate of charge carriers in photoexcited solar materials, they are mostly successful when it comes to charge localization (by small polaron formation either at defects or at regular sites) because of the strong signals generated by an oxidation state change of elements. However, charge localization is only one aspect of the charge dynamics and therefore, studies using optical methods $(\mathrm{THz}$ to the visible range) are needed. ${ }^{[32]}$ As these probe free charge carriers, they are not material specific. Therefore, the recent implementation ${ }^{[33,34]}$ of a deep-ultraviolet probe that interrogates the excitonic transitions of transition metal oxides (that all lie below $380 \mathrm{~nm}$ ) upon electron delivery to the $\mathrm{CB}$ of the materials, offers an ideal complement to the X-ray and optical approaches.

Finally, this review focussed on studies with typically $70-100$ ps resolution, but studies at higher (femtosecond) time resolution provide detailed insight into the dynamics of charge carriers and their trapping as recently demonstrated by fs-XAS studies of $\mathrm{TiO}_{2}$ nanoparticles. ${ }^{[10]}$ In particular, the high photon fluxes and femtosecond temporal resolution offered by hard $\mathrm{X}$-ray free electron lasers inevitably deliver new insight. ${ }^{23]}$ The Swiss Free Electron Laser (SwissFEL) ${ }^{[35,36]}$ is coming into operation at the end of 2017, almost simultaneous to the launch of the European XFEL in Hamburg[37] and the Korean XFEL (PAL). ${ }^{[38]}$ These machines along with the already existing ones (LCLS in Stanford$\mathrm{USA}^{[39]}$ and SACLA in Japan ${ }^{[40]}$ ) and others still in construction (the Shanghai $\mathrm{X}$-ray free electron laser, SFEL[41]) make the future of experiments on solar materials very promising.

\section{Acknowledgments:}

We acknowledge financial support from the Swiss NSF via the NCCR:MUST and contracts $n^{\circ}$ 206021_157773, 20020_153660 and 407040_154056 (PNR 70) and the European Research Council Advanced Grants H2020
ERCEA 695197 DYNAMOX. We thank the technical staff at beamlines PHOENIX, MicroXAS and SuperXAS of the Swiss Light Source for their crucial support. We also thank Prof. M. Kovalenko and his team (ETHZ) for providing us the perovskite samples.

\section{Received: August 21, 2017}

[1] H. Zhou, Q. Chen, G. Li, S. Luo, T.-b. Song, H.-S. Duan, Z. Hong, J. You, Y. Liu, Y. Yang, Science 2014, 345, 542.

[2] S. D. Stranks, G. E. Eperon, G. Grancini, C. Menelaou, M. J. Alcocer, T. Leijtens, L. M. Herz, A. Petrozza, H. J. Snaith, Science 2013, $342,341$.

[3] L. M. Herz, ACS Energy Lett. 2017, 2, 1539.

[4] A. Miyata, A. Mitioglu, P. Plochocka, O. Portugall, J. T.-W. Wang, S. D. Stranks, H. J. Snaith, R. J. Nicholas, Nat. Phys. 2015, 11, 582.

[5] G. Nedelcu, L. Protesescu, S. Yakunin, M. I. Bodnarchuk, M. J. Grotevent, M. V. Kovalenko, Nano Lett. 2015, 15, 5635

[6] J. Endres, D. A. Egger, M. Kulbak, R. A. Kerner, L. Zhao, S. H. Silver, G. Hodes, B. P. Rand, D. Cahen, L. Kronik, J. Phys.Chem. Lett. 2016, 7 , 2722.

[7] M. Z. Liu, M. B. Johnston, H. J. Snaith, Nature 2013, 501, 395.

[8] C. S. Ponseca, T. J. Savenije, M. Abdellah, K. Zheng, A. Yartsev, T. Pascher, T. Harlang, P. Chabera, T. Pullerits, A. Stepanov, J.-P. Wolf, V Sundström, J. Am. Chem. Soc. 2014, 136, 5189.

[9] M. H. Rittmann-Frank, C. J. Milne, J. Rittmann, M. Reinhard, T. J. Penfold, M. Chergui, Angew. Chem. Int. Ed. 2014, 53, 5858.

[10] F. G. Santomauro, A. Lubcke, J. Rittmann, E. Baldini, A. Ferrer, M. Silatani, P. Zimmermann, S. Grubel, J. A. Johnson, S. O. Mariager, P. Beaud, D. Grolimund, C. Borca, G. Ingold, S. L. Johnson, M. Chergui, Sci. Rep. UK 2015, 5 , 14834.

[11] D. C. Koningsberger, R. Prins, 'X-ray absorption : principles, applications, techniques of EXAFS, SEXAFS, and XANES' Wiley, New York, 1988.

[12] C. Bressler, M. Chergui, Chem. Rev. 2004, 104, 1781.

[13] M. Chergui, Acta Crystallogr, A 2010, 66, 229.

[14] C. J. Milne, T. J. Penfold, M. Chergui, Coord. Chem. Rev. 2014, 277, 44.

[15] F. A. Lima, C. J. Milne, D. C. V. Amarasinghe, M. H. Rittmann-Frank, R. M. van der Veen, M. Reinhard, V. T. Pham, S. Karlsson, S. L. Johnson, D. Grolimund, C. Borca, T. Huthwelker, M. Janousch, F. van Mourik, R. Abela, M. Chergui, Rev. Sci. Instr. 2011, 82, 063111.

[16] M. Silatani, F. A. Lima, T. J. Penfold, J. Rittmann, M. E. Reinhard, H. M. RittmannFrank, C. Borca, D. Grolimund, C. J. Milne, M. Chergui, Proc. Natl. Acad. Sci. USA 2015, 112, 12922.

[17] V. Luca, J. Phys. Chem. C 2009, 113, 6367.

[18] H. Z. Zhang, B. Chen, J. F. Banfield, G. A Waychunas, Phys. Rev. B 2008, 78, 214106.

[19] F. Farges, G. E. Brown, J. J. Rehr, Phys. Rev. B 1997, 56, 1809.

[20] T. Uozumi, K. Okada, A. Kotani, O. Durmeyer, J. P. Kappler, E. Beaurepaire, J. C. Parlebas, Europhys. Lett. 1992, 18, 85.

[21] E. L. Shirley, J. Elec. Spect. Rel. Phenom. 2004, $136,77$.

[22] T. L. Hanley, V. Luca, I. Pickering, R. F. Howe, J. Phys. Chem. B 2002, 106, 1153.

[23] Y. Obara, H. Ito, T. Ito, N. Kurahashi, S. Thürmer, H. Tanaka, T. Katayama, T. Togashi, S. Owada, Y.-i. Yamamoto, S. Karashima, J.
Nishitani, M. Yabashi, T. Suzuki, K. Misawa, Struct. Dynam. 2017, 4, 044033.

[24] F. G. Santomauro, J. Grilj, L. Mewes, G. Nedelcu, S. Yakunin, T. Rossi, G. Capano, A. A. Haddad, J. Budarz, D. Kinschel, D. S. Ferreira, G. Rossi, M. G. Tovar, D. Grolimund, V. Samson, M. Nachtegaal, G. Smolentsev, M. V. Kovalenko, M. Chergui, Struct. Dynam. 2017, 4, 044002.

[25] C. G. Elles, I. A. Shkrob, R. A. Crowell, D. A Arms, E. C. Landahl, J. Chem. Phys. 2008, 128 , 061102.

[26] V. T. Pham, W. Gawelda, Y. Zaushitsyn, M. Kaiser, D. Grolimund, S. L. Johnson, R. Abela, C. Bressler, M. Chergui, J. Am. Chem. Soc. 2007, 129, 1530.

[27] V. T. Pham, T. J. Penfold, R. M. van der Veen, F. Lima, A. El Nahhas, S. L. Johnson, P. Beaud, R. Abela, C. Bressler, I. Tavernelli, C. J. Milne, M. Chergui, J. Am. Chem. Soc. 2011, 133, 12740.

[28] J. C. Swarbrick, U. Skyllberg, T. r. Karlsson, P. Glatzel, Inorg. Chem. 2009, 48, 10748.

[29] S. Hunsche, K. Leo, H. Kurz, K. Köhler, Phys. Rev. B 1994, 49, 16565 .

[30] K. Zheng, M. Abdellah, Q. Zhu, Q. Kong, G. Jennings, C. A. Kurtz, M. E. Messing, Y. Niu, D. J. Gosztola, M. J. Al-Marri, X. Zhang, T. Pullerits, S. E. Canton, J. Phys. Chem. Lett. 2016, 7, 4535.

[31] T. J. Penfold, J. Szlachetko, F. Santomauro, A. Britz, W. Gawelda, G. Doumy, A.-M. March, S. Southworth, J. Rittmann, R. Abela, M. Chergui, C. J. Milne, Nat. Commun. 2015, under review.

[32] C. S. Ponseca, P. Chábera, J. Uhlig, P. Persson, V. Sundström, Chem. Rev. 2017, 117, 10940.

[33] E. Baldini, L. Chiodo, A. Dominguez, M. Palummo, S. Moser, M. Yazdi-Rizi, G. Auböck, B. P. Mallett, H. Berger, A. Magrez, Nat. Commun. 2017, 8, 13.

[34] E. Baldini, T. Palmieri, T. Rossi, M. Oppermann, E. Pomarico, G. Auböck, M. Chergui, J. Am. Chem. Soc. 2017, 139, 11584 .

[35] C. J. Milne, T. Schietinger, M. Aiba, A. Alarcon, J. Alex, A. Anghel, V. Arsov, C. Beard, P. Beaud, S. Bettoni, Appl. Sci. 2017, 7, 720.

[36] M. Chergui, P. Beaud, J. A. van Bokhoven, Th. Feurer, J. Haase, G. Ingold, S. L. Johnson, G. Knopp, H. Lemke, C. J. Milne, L. Patthey, B. Pedrini, P. Radi, G. Schertler, J. Standfuss, U. Staub, Struct. Dyn. 2017, 4.

[37] T. Tschentscher, C. Bressler, J. Grünert, A Madsen, A. Mancuso, M. Meyer, A. Scherz, H. Sinn, U. Zastrau, Appl. Sci. 2017, 7, 592.

[38] I. Ko, H.-S. Kang, H. Heo, C. Kim, G. Kim, C.K. Min, H. Yang, S. Baek, H.-J. Choi, G. Mun, B. Park, Y. Suh, D. Shin, J. Hu, J. Hong, S. Jung, S.-H. Kim, K. Kim, D. Na, S. Park, Y. Park, Y. Jung, S. Jeong, H. Lee, S. Lee, S. Lee, B. Oh, H. Suh, J.-H. Han, M. Kim, N.-S. Jung, Y.-C. Kim, M.-S. Lee, B.-H. Lee, C.-W. Sung, I.-S. Mok, J.-M. Yang, Y. Parc, W.-W. Lee, C.-S. Lee, H. Shin, J. Kim, Y. Kim, J. Lee, S.-Y. Park, J. Kim, J. Park, I. Eom, S. Rah, S. Kim, K. H. Nam, J. Park, J. Park, S. Kim, S. Kwon, R. An, S. Park, K. Kim, H. Hyun, S. Kim, S. Kim, C.-J. Yu, B.S. Kim, T.-H. Kang, K.-W. Kim, S.-H. Kim, H.S. Lee, H.-S. Lee, K.-H. Park, T.-Y. Koo, D.-E. Kim, K. Lee, Appl. Sci. 2017, 7, 479.

[39] R. Schoenlein, S. Boutet, M. Minitti, A. M. Dunne, Appl. Sci. 2017, 7, 850.

[40] M. Yabashi, H. Tanaka, K. Tono, T. Ishikawa, Appl. Sci. 2017, 7, 604.

[41] Z. Zhao, D. Wang, Q. Gu, L. Yin, M. Gu, Y. Leng, B. Liu, Appl. Sci. 2017, 7, 607. 